\title{
PLANETARY AND EXOPLANETARY STUDIES WITH THE GIANT RADIO TELESCOPE LOFAR
}

\author{
P. Zarka*, J. N. Girard*, J.-M. Grießmeier ${ }^{\dagger}$, S. Hess ${ }^{\ddagger}$, and L. Denis ${ }^{\S}$
}

\begin{abstract}
The european radio telescope LOFAR is in its commissioning phase. It is a huge interferometer operating at low frequencies (30 to $240 \mathrm{MHz}$ ), composed of $\sim 50$ phased arrays (or stations) which are themselves gathering 864 to 1632 antennas. $\sim 40$ stations are located in the Netherlands along with the central correlator, and the other stations are spread over surrounding countries.In France, one of these arrays (1632 elements and their 96 associated receptors) has been built at the Nançay radioastronomy observatory.

LOFAR will operate as an interferometer (for spectro-imagery) and as a global coherent phased array (for dynamic spectrometry).Its planetary objectives, that will be addressed by the Planets Working Group of LOFAR's "Transients" Key Science Project, include the fast imaging of the Jovian magnetosphere through its auroral radio emissions, and the detection and study of lightning on every planet of our solar system.

Jupiter is the most intense low-frequency point source in the sky $\left(>10^{6} \mathrm{Jy}\right)$, allowing extensive commissioning tests, which will be briefly described (including early interferometric observations between Nançay and the Netherlands). In the case of Saturn's lightning, the large instantaneous bandwidth (up to $48 \mathrm{MHz}$ ) and the high time resolution of LOFAR (down to $5 \mu s$ per spectrum) allow us to measure the discharge spectrum and energy(see Grießmeier et al., this issue, 145-154).

Moreover, one of the major objectives of LOFAR is the detection and study of exoplanetary radio emissions, which will be an unique source of information about the magnetic field, rotation and inclination of these objects, and about planet-star plasma interactions in general. In our solar system, each planetary magnetosphere has common as well as singular characteristics, which make it differ from the others. Radio detection of exoplanetary magnetospheres with allow us to explore parameter space of magnetospheric physics and enhance comparative studies (see Zarka, this issue, 287-301).
\end{abstract}

* LESIA, Observatoire de Paris, UMR CNRS 8109, 92195 Meudon, France

† Laboratoire de Physique et Chimie de l'Environment et de l'Espace, CNRS, 45071 Orléans, France

$\ddagger$ Laboratory for Atmospheric and Space Physics, University of Colorado, Boulder, CO 80303-7814, USA

§ Station de Radioastronomie, Observatoire de Paris, CNRS, Nançay, France 
